\title{
¿BIENVENIDO, MÍSTER MARSHALL! LA AYUDA ECONÓMICA AMERICANA Y LA ECONOMÍA ESPAÑOLA EN LA DÉCADA DE 1950
}

\author{
ÓSCAR CALVO GONZÁLEZ *
}

London School of Economics

\section{RESUMEN}

¿Cuáles fueron los efectos que la ayuda americana tuvo en la economía española de los años cincuenta? Este artículo afronta esta pregunta desde una perspectiva alejada de los enfoques que, enfatizando el desahogo de embotellamientos productivos, habian sido predominantes hasta la fecha en la historiografía económica española. Se elabora aquí una interpretación que sugiere que la concesión de la ayuda americana pudo tener, entre sus consecuencias, la rehabilitación de las expectativas empresariales privadas. El programa de ayuda americano, como parte del proceso de aceptación internacional del régimen de Franco, pudo generar una mejora de la credibilidad política del régimen que se tradujo en un clima de mayor seguridad y confianza de los agentes económicos, un aumento de la inversión y, con ella, del crecimiento económico. El artículo concluye con una llamada a profundizar la investigación sobre este episodio histórico.

\section{ABSTRACT}

What was the impact of American aid to Spain during the 1950s? This article revisits this question, addressing it from a rather different perspective to the focus on the relief of supply bottlenecks that dominates the existing Spanish economic historiography. It suggests that there is a prima facie case for the aid programme to have had a positive effect on private agents' expec-

* Me gustaría agradecer, sin implicar en la interpretación y errores del texto, los comen. tarios de María Jesús Asensio, Pedro Fraile, Fernando Guirao, Pablo Martín Aceña, Leandro Prados de la Escosura, José María Serrano Sanz y, especialmente, de Nick Crafts. Igualmente doy las gracias por las sugerencias y comentarios de los participantes en seminarios en la London School of Economics, Universidad Carlos III de Madrid y UIMP.Cuenca, así como de los dos evaluadores anónimos de esta revista. 
tations. The American backing, as part of the process of international acceptance of the Franco regime, may have provided a «commitment technology» that solved problems of credibility, which would have otherwise hindered investment and hence the resumption of economic growth. The paper concludes with an agenda for further research.

\section{INTRODUCCIÓN}

En 1952, el cineasta español García Berlanga estrenaba Bienvenido, Míster Marshall con gran éxito de público y crítica. La película, que se centra en las esperanzas de los habitantes de un pueblo de recibir el maná americano, habría de ser profética, no sólo de la firma de los acuerdos entre España y los Estados Unidos en septiembre de 1953, sino también de cómo ha visto cierta literatura el impacto de la ayuda americana en la economía española. Así, historiadores políticos, centrados en aspectos diplomáticos, tradicionalmente mencionan los escasos efectos económicos para resaltar que lo limitado del programa de ayuda americana constituye una prueba más del desequilibrio de unos acuerdos que reflejan la «evidente debilidad» del régimen de Franco ${ }^{1}$. Si en la película de García Berlanga los americanos pasan de largo sin detenerse, esta corriente en la historiografía apunta que la ayuda americana significó «muy poco» para el desarrollo económico español, y enfatiza que los montantes de ayuda hechos efectivos fueron decepcionantemente limitados ${ }^{2}$.

La historiografía económica, por su parte, ha sido generalmente más optimista sobre las consecuencias de la ayuda americana. Se apunta que tuvo efectos multiplicadores, o que alivió los cuellos de botella en la producción, aunque sin realizar un análisis cuantitativo de dichos mecanismos de transmisión ${ }^{3}$. Significativamente, la reciente historiografía específica sobre el programa de apoyo americano ha corrido a cargo de historiadores políticos, lo cual contrasta con la falta de revisión de este tema en el marco de la historia económica ${ }^{4}$. ¿Es pertinente semejante revisión?

${ }^{\prime}$ Fusi (1995), p. 141. Viñas et al. (1979), pp. 793 ss.

2 Tamames (1986), p. 222. Más recientemente, Guirao (1996), p. 91; Viñas (1981a), p. 315 .

${ }^{3}$ Sardá (1978); Clavera et al. (1978); Fontana y Nadal (1976), p. 513; Shearer (1959); Harrison (1978), p. 154, y García Delgado (1986).

4 Jarque Íñiguez (1998), Edwards (1999) y Liedtke (1998). Este último autor es explicitamente revisionista enfatizando, desde el estudio de fuentes americanas, la urgencia de los Estados Unidos por llegar a un acuerdo lo que confirió a España un poder de negociación 
En primer lugar, conviene resaltar el protagonismo que la década de 1950 (que coincide con la ayuda americana) ha alcanzado en la historiografía. Los Estados Unidos habían comenzado a conceder ayuda económica al gobierno español desde el agravamiento de la guerra fría en 1950 , así como a promover la aceptación internacional del régimen con medidas como el retorno unilateral de su embajador a Madrid. Este creciente apoyo al régimen de Franco llevó finalmente a la firma de los acuerdos de 26 de septiembre de 1953, conocidos como el Pacto de Madrid. Dada la coincidencia del apoyo americano con la revitalización del crecimiento económico en España, resulta relevante preguntarse por la existencia y naturaleza de los posibles nexos causales entre ambos acontecimientos ${ }^{5}$.

La revisión de este tema es, además, oportuna por el desarrollo de la historiografía sobre el impacto de otros programas de ayuda extranjera, notablemente sobre el Plan Marshall ${ }^{6}$. Tras el revisionismo de mediados de los años ochenta que puso de manifiesto la escasa contribución directa del Plan Marshall a través de la balanza comercial, la discusión ha pasado a centrarse en los efectos indirectos que aspectos tales como la condicionalidad asociada a la ayuda pudieron tener sobre las economías receptoras ${ }^{7}$.

Este artículo pretende realizar, en primer lugar, un breve resumen del estado de la cuestión en la bibliografía. En segundo lugar se sugiere la hipótesis de que la concesión de la ayuda americana trajo consigo una rehabilitación de las expectativas empresariales, que se tradujo en un aumento de la inversión, y con ella del crecimiento económico. El que la confianza empresarial mejorase durante los años cincuenta no es una tesis en sí novedosa; sin embargo, la conexión con el episodio de ayuda americana no ha sido explorada en detalle hasta ahora, por lo que el artículo se cierra con una llamada a profundizar la investigación sobre este aspecto del impacto de la ayuda americana en la economía española ${ }^{8}$.

mayor del habitualmente reconocido en la historiografía. Liedtke (1999) también se centra en el Pacto de Madrid.

'La tasa media de crecimiento anual del producto real per capita durante los años cincuenta fue del 4,7 por 100 comparada con el 1,2 por 100 durante los cuarenta, Prados de la Escosura (1995).

- La efectividad de la ayuda extranjera al desarrollo es también objeto de creciente atención por parte de los organismos internacionales; véase, por ejemplo, Banco Mundial (1998).

7 Milward (1984); Eichengreen y Uzan (1992), y De Long y Eichengreen (1993).

* Fraile (1999), p. 240; Spitäller y Galy (1992), p. 2; González (1989/1990) es el más claro precursor de estas ideas. 


\section{INTERPRETACIONES TRADICIONALES SOBRE EL IMPACTO ECONÓMICO DE LA AYUDA AMERICANA}

Dos son los efectos de la ayuda americana sobre los que la historiografía se ha pronunciado con mayor insistencia. En primer lugar, se argumenta que la ayuda permitió la importación de bienes «indispensables» para el tejido productivo español, desahogando así numerosos cuellos de botella en la producción ${ }^{9}$. El segundo efecto hace referencia a la influencia de los Estados Unidos en la política española ${ }^{10}$. Examinemos estos argumentos por separado, repasando, en primer lugar, los valores de las mercancías recibidas con cargo a la ayuda americana (Cuadros 1 y 2 ).

La ayuda acumulada supuso algo menos de un 1 por 100 del PIB español en el período 1953-1963 ${ }^{11}$. No sólo es ésta una cantidad inferior a las recibidas por los países receptores del Plan Marshall, sino que, dado el fuerte crecimiento del PIB, sólo supuso una contribución al crecimiento de éste de un 3,5 por $100^{12}$. Igualmente, el Cuadro 1 muestra cómo en su mayor parte, sobre todo en los primeros años, la ayuda fue destinada a compras de inputs o alimentos, no tanto a la importación de nueva maquinaria que mejorase la capacidad productiva de la economía española, una queja habitual por parte española ${ }^{13}$. Haciendo uso de los datos anuales para el período 1954-1958, en el que se concentran los desembolsos de ayuda, observamos cómo la media de la contribución de la ayuda al incremento del PIB fue de tan sólo un 6,5 por 100 anual. De hecho, lo limitado del programa de ayuda generó un descontento creciente en el gobierno español ${ }^{14}$. Los Cuadros 1 y 2 también destacan los considerables retrasos

${ }^{9}$ García Delgado (1986), p. 292; Harrison (1985), pp. 133-134.

10 Preston (1993), p. 624. Véase Fanjul (1981) para una síntesis del tratamiento de estos dos aspectos.

${ }^{11} 0,80$ por 100 en $1950-1959$; 0,95 por 100 para $1953-1963$; y 0,98 por 100 para los años de mayor intensidad de la ayuda, 1954-1958. Se ha utilizado el tipo de cambio medio de las importaciones, Serrano Sanz y Asensio (1997), para reflejar el verdadero desahogo que supusieron para la balanza comercial. En éste, como en sucesivos cálculos, la estimación del PIB ha sido tomada de Prados de la Escosura (1995).

${ }^{12}$ Ayuda desembolsada como porcentaje del incremento del PIB del período. Para los países del Plan Marchall, la media es del 2,5 por 100 del PIB aproximadamente; De Long y Eichengreen (1993).

${ }_{13}$ Baldrich (1957). Además, en ocasiones, partidas autorizadas para un tipo de bien eran posteriormente dedicadas a la importación de otros bienes, lo que explica las discrepancias entre los valores autorizados y desembolsados en el Cuadro 1.

${ }^{14}$ En la prensa véase, por ejemplo, Ya de 6 de febrero de 1958 , o $A B C$ de 23 de junio de 1963 , que destaca que España había recibido apenas 3,75 dólares por habitante 


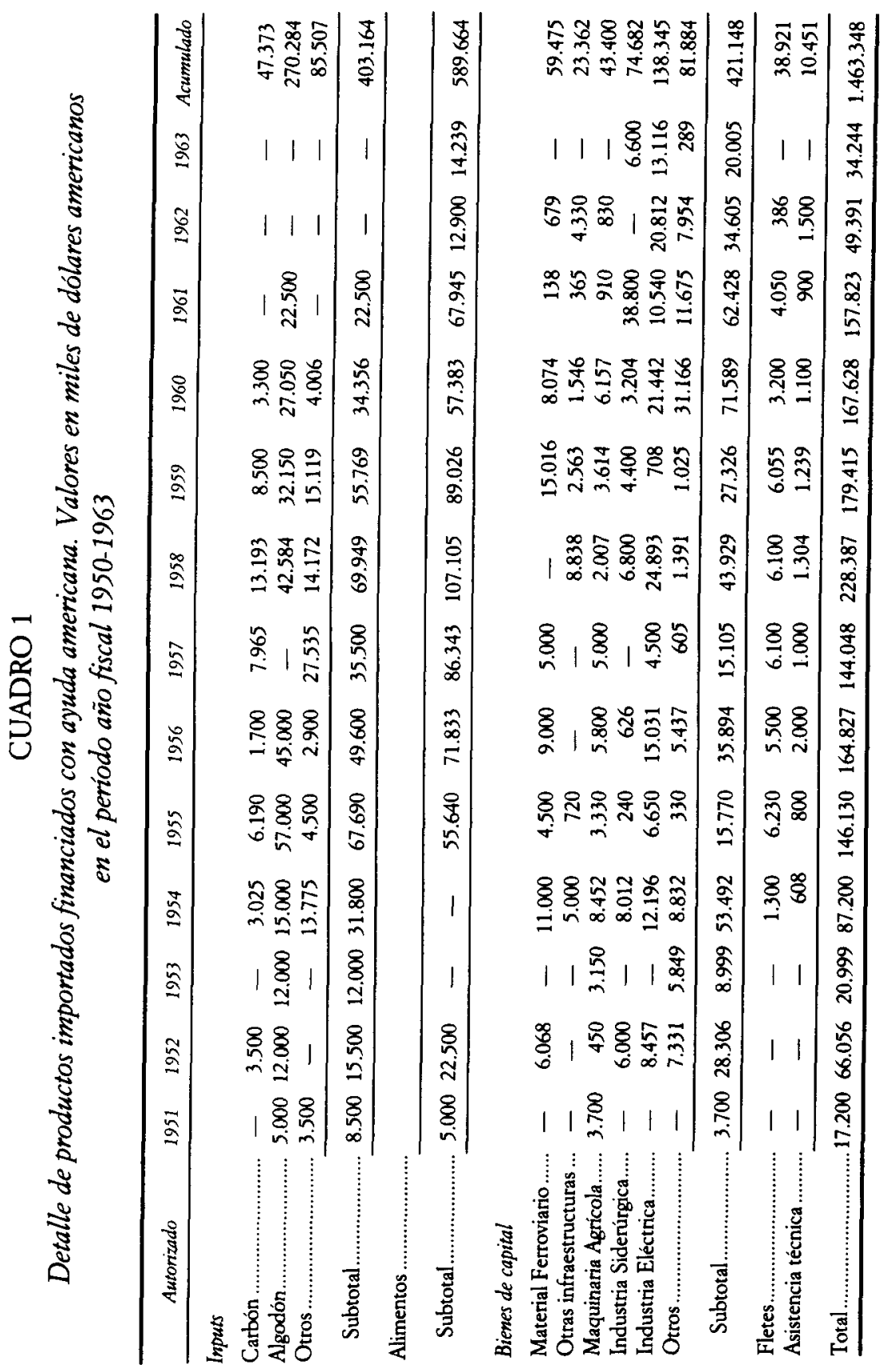




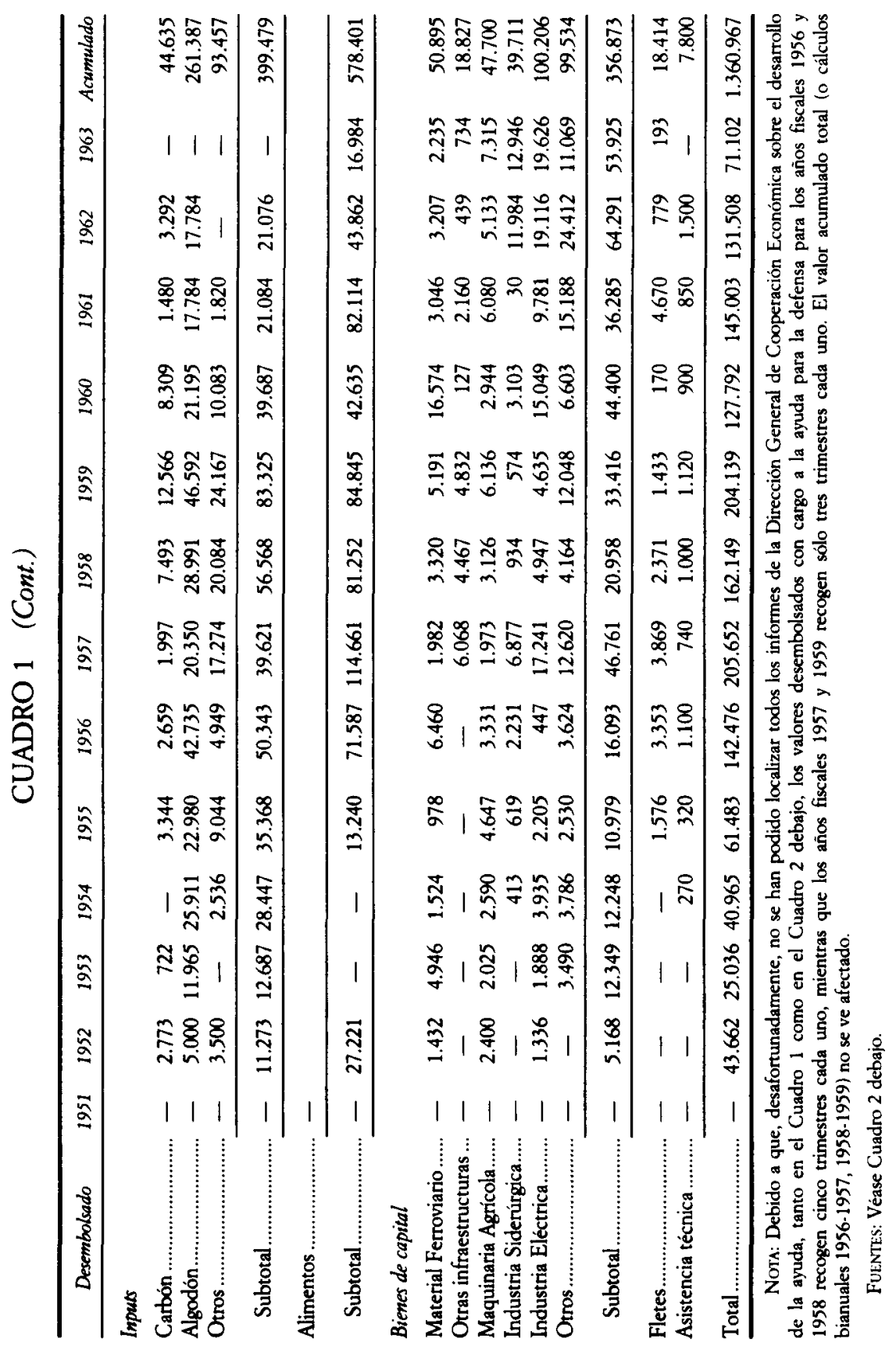




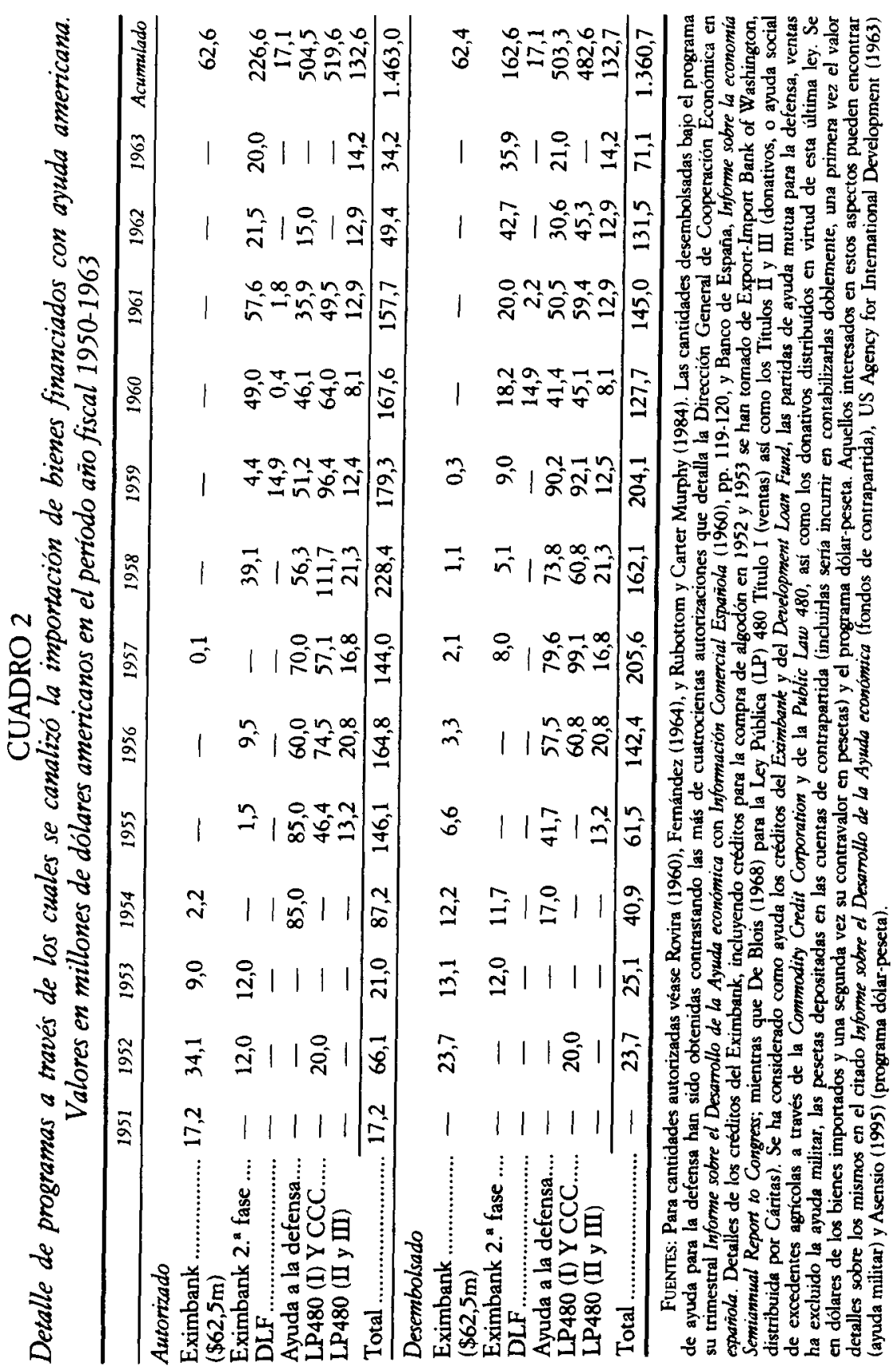


entre el momento en que las partidas eran autorizadas y su llegada a España, lo que pone de manifiesto que la ayuda no sólo no empezó a agotarse en los últimos años de los cincuenta, sino que registró su mayor cuantía en 1957-1959. La ayuda americana tampoco permitió un incremento apreciable de la intensidad importadora ${ }^{15}$. Así, el grado de apertura de la economía española durante los años cincuenta se mantuvo en niveles muy bajos, situándose el máximo en 1953; es decir, con anterioridad a que la ayuda permitiese importaciones sustanciales ${ }^{16}$.

Se ha sugerido, sin embargo, que estas importaciones suplementarias que la ayuda americana permitía tuvieron un efecto multiplicador:

«[t]eniendo en cuenta los amplios estrangulamientos que atenazaban la economía española. Un incremento en las importaciones, aunque no tuviera gran entidad en términos absolutos, había de tener efectos immediatos sobre el nivel de actividad interior, siempre que dichas importaciones se dirigiesen hacia aquellos sectores en que sus ofertas eran más limitativas» ${ }^{17}$.

Este argumento se presta al uso del análisis input-output para cuantificar la importancia de los efectos directos e indirectos de esas importaciones adicionales. Basándonos en la metodología habitual de cálculo de encadenamientos productivos, podemos obtener los encadenamientos para los 28 sectores de la tabla input-output de $1954^{18}$. El primer resultado llamativo es el gran número de importaciones financiadas con la ayuda que recayeron en bienes y sectores de bajos encadenamientos productivos, lo cual fue debido, en gran medida, al énfasis que los Estados Unidos pusieron en incrementar las ventas de productos agrícolas y alimentos. Las propias auto-

y año. Este descontento «popular» era uno de los argumentos utilizados por el Ministerio de Asuntos Exteriores para presionar a los Estados Unidos en la renegociación de los acuerdos. Nota sin firmar titulada «Economía española y ayuda americana», MAE, Leg. 4615, Exp. 15.

15 El ratio importaciones/PIB se estanca por debajo del 8 por 100 en el período 1955-1958, paradójicamente cuando la ayuda fue más cuantiosa. Cálculos con datos tomados de Martínez (1998).

${ }^{16} \mathrm{El}$ grado de apertura (11 por 100 en 1953) medido como la suma de importaciones y exportaciones dividido por el PIB, se ha obtenido utilizando los diferentes tipos de cambios para importaciones y exportaciones obtenidos por Serrano Sanz y Asensio (1997) y datos de Martínez (1998) para el comercio exterior. Estimaciones anteriores, Tena (1989), muestran un estancamiento de las importaciones en términos reales durante 1953-1958.

${ }_{17}$ Clavera et al. (1978), p. 254.

is Yotopoulos y Nugent (1973). Una estimación del encadenamiento total para el sector j se obtiene sumando los $i$ elementos de la columna $j$ en la matriz inversa de Leontief. La tabla input-output de 1954, en Instituto de Estudios Políticos (1958). 
ridades españolas llegaron a lamentarse del «poco o nulo interés que tienen las nuevas ofertas sobre excedentes agrícolas» ${ }^{19}$. Algodón, trigo o carbón, tenían todos ellos multiplicadores inferiores a la media ${ }^{20}$.

Podría argumentarse que, pese a que las importaciones financiadas directamente con la ayuda no tuviesen grandes efectos multiplicadores, la ayuda americana permitió liberar divisas que, éstas sí, se dedicaron a importar aquellos bienes con mayores encadenamientos. Utilizando el valor medio de encadenamientos totales, y así captar este argumento, para el período 1954-1958, obtenemos que la contribución directa e indirecta debida a las importaciones financiadas con ayuda americana sería no del 6,5 por 100 , sino del 10,5 por 100 del crecimiento del PIB. En otras palabras, la economía española habría podido crecer al 90 por 100 del ritmo con que lo hizo en un contrafactual en el que no se hubiese recibido ayuda. Estos cálculos, aunque a vuela pluma, son indicativos del limitado efecto de la ayuda a través de efectos multiplicadores. Además, es preciso recordar que los limitativos supuestos del modelo input-output, descartando una posible sustitución de inputs, tienden a exagerar la contribución de la ayuda.

El segundo efecto del programa americano de ayuda al que la historiografía suele referirse es la influencia en la política económica española que los Estados Unidos obtienen a raíz del acuerdo. Se argumenta que el programa de política económica relativamente reformista «no hubiera podido ser ni siquiera esbozado sin contar con esta ayuda» ${ }^{21}$. Aunque ciertamente este estímulo supuso un apoyo en el largo plazo para aquellos elementos más liberalizadores dentro del gabinete, sus efectos no deben sobrevalorarse ${ }^{22}$. No sólo las medidas liberalizadoras fueron extremadamente graduales, sino que el gobierno americano realizó una presión muy tímida, con demandas en muchos casos retóricas ${ }^{23}$. La inclusión de com-

19 Carta del Embajador Areilza al Ministro de Comercio Arburúa, Washington, D. C., 31 de diciembre de 1954. MAE, Leg. 4615, Exp. 15.

20 1,283 para algodón; 1,356 para cereales; 1,439 para carbón. Asumiendo que no existen posibilidades de sustitución de inputs, estos valores significan que, por ejemplo, el carbón producido permite que otras actividades lo utilicen como input, con lo que el valor total de la producción que una peseta de carbón permite es en realidad 1,439 pesetas. La media (no ponderada) de los encadenamientos totales de los 28 sectores es 1,635.

${ }^{21}$ Clavera et al. (1978), p. 255.

${ }^{22}$ Harrison (1985), p. 134.

${ }^{23}$ Anderson (1970), González (1979). Pese a la liberalización de algunos precios en 1951, sobre todo agrarios, en muchas áreas de la política económica las restricciones continuaron siendo especialmente rígidas. Véase Buesa (1984) para la política industrial. La política comercial, por su parte, se caracterizó por un cambio de rumbo «lento y exas- 
promisos reformistas en el texto de los acuerdos fue vaga y sin mecanismos vinculantes ${ }^{24}$. La propia historiografía que enfatiza la influencia americana en el cambio de política económica reconoce que «no pueden ser detectados esfuerzos relevantes para exigir del Gobierno español una mayor liberalización de su política económica» ${ }^{25}$. Un claro ejemplo fueron los desembolsos de los fondos de contrapartida que, aunque requerían el consentimiento de los Estados Unidos, no fueron utilizados como medida de presión para forzar la adopción de reformas en la política económica ${ }^{26}$. La historiografía sobre el Plan Marshall sugiere que en ese caso los Estados Unidos sí intentaron ejercer tal presión, aunque sin éxito ${ }^{27}$. En cierto sentido, en España no había lugar a ese forcejeo dado el alto porcentaje de los fondos de contrapartida que se había acordado fuesen destinados a la construcción de las bases ( 70 por 100 de la contrapartida de apoyo para la defensa hasta 1958) ${ }^{28}$. Tampoco la firma de los acuerdos hispano-americanos supuso una masiva entrada de capital americano en España. Habrá que esperar, pese a las quejas testimoniales de los americanos al respecto, al Plan de Estabilización y Liberalización de 1959 para que la economía española se reabra a la inversión extranjera directa ${ }^{29}$. En resumen, como señala Guirao, los Estados Unidos no sólo no presionaron para obtener el cumplimiento de lo acordado en materia de política económica, sino que rechazaron las solicitudes españolas de apoyo financiero para hacer frente a los compromisos de una posible entrada en la Unión

perantemente pausado» según Viñas et al. (1979), p. 743. Sobre la presión americana, véanse, por ejemplo, las cartas del Embajador Lodge al Ministro de Asuntos Exteriores Martín Artajo fechadas en Madrid los días 5 de octubre y 23 de noviembre de 1956, en las que el representante norteamericano se muestra más interesado en conseguir que España compre más excedentes agrícolas que en asegurar la adopción de determinadas medidas económicas. MAE, Leg. 7741, Exp. 3.

${ }^{24}$ Tacconi (1955), Sause (1957) y Fernández (1964), para un recorrido por los compromisos adquiridos en materia de política económica por el gobierno español en el Pacto de Madrid.

${ }^{25}$ Clavera et al. (1978), p. 256.

${ }^{26}$ Los fondos de contrapartida ni se mencionaban en las ocasiones en que los Estados Unidos sugerían medidas contra la inflación o mayores libertades para el capital extranjero. Véase nota 23 arriba.

${ }^{27}$ Esposito (1994).

${ }^{2 \times}$ Fernández (1964), p. 24.

${ }^{29}$ Sáinz Moreno (1965). El informe del Stanford Research Institute (1972) da cifras muy significativas sobre el valor total del stock de inversiones americanas en España, que pasaron de 40 millones de dólares en 1953 a 50 millones en 1959 y a 275 millones en 1965. 
Europea de Pagos y la Organización Europea para la Cooperación Económica ${ }^{30}$.

Así pues, este recorrido por los argumentos habituales en la historiografía sobre el impacto de la ayuda americana no parece sino confirmar el pesimismo de ciertos autores y justificar el abandono de este tema por los investigadores. Alternativamente, cabe sugerir que estos enfoques no reflejan un importante aspecto de la ayuda. La siguiente sección del artículo se dedica a elaborar una justificación teórica de un nuevo enfoque para analizar la pregunta sobre los efectos de la ayuda americana en la economía española.

\section{UNA HIPÓTESIS DE CREDIBILIDAD (I): FUNDAMENTO TEÓRICO}

Uno de los aspectos tratados por la reciente historiografía sobre el efecto de la ayuda extranjera en el crecimiento económico europeo de posguerra, es el papel desempeñado por aquélla en contribuir a un clima más favorable a la inversión ${ }^{31}$. En concreto, se sugiere que el desembolso de la ayuda americana condicionado a la puesta en marcha de instituciones como la Unión Europea de Pagos empujó a los países de Europa Occidental hacia una cooperación internacional que hubiese resultado difícil de alcanzar sin mediar el incentivo de la ayuda americana ${ }^{32}$. Este tipo de argumento se presta a ser tratado de manera algo más formal (utilizando la teoría de juegos simple).

Analicemos la interacción entre el gobierno, por un lado, y los agentes económicos privados, por otro. Estos últimos deciden si invertir o no (decisión tomada en $t=0$ y vinculante hasta el final del período $t=1$ ). Supongamos que la producción $(\mathrm{Y})$ en el periodo $\mathrm{t}=1$ es una función (entre otros factores) de la decisión de invertir en el periodo $t=0, y$ así $Y=100$ si los agentes deciden no invertir, mientras que $Y=200 \mathrm{si}$ los agentes invierten (incurriendo un coste igual a 50). El gobierno decide a continuación si llevar a cabo políticas confiscatorias (quizás resultado de un cambio de régimen) o, alternativamente, imponer una carga fiscal

\footnotetext{
30 Guirao (1996), p. 92.

${ }^{31}$ Eichengreen (1996) y Berger y Ritschl (1995).

32 Eichengreen (1993).
} 
que no desincentive a los inversores (por seguir con un ejemplo sencillo, del 0 por 100) ${ }^{33}$. El siguiente Diagrama 1 refleja dicho juego.

\section{DIAGRAMA 1}

Juego entre agentes económicos privados y gobierno

(Rendimientos para los agentes económicos privados en primer lugar)

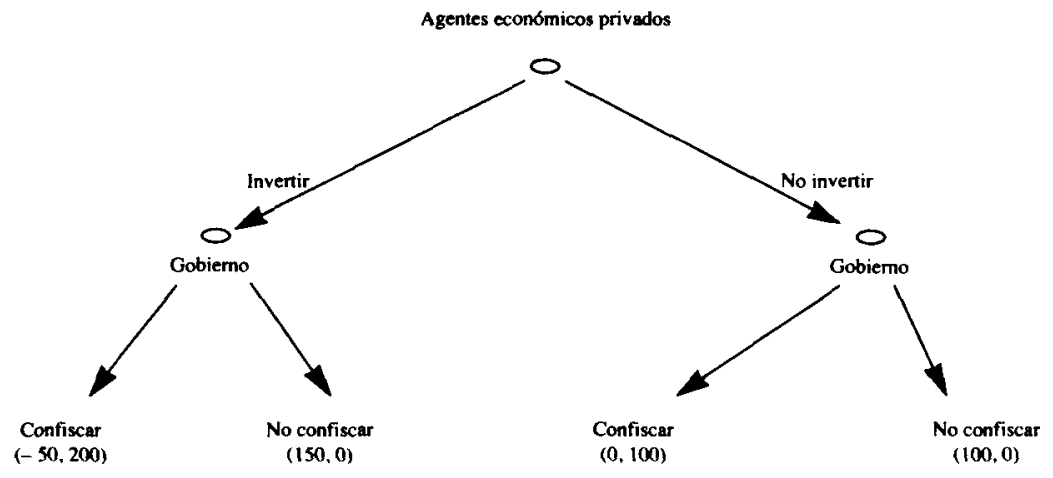

El gobierno tiene una única estrategia dominante, «confiscar», ya que el rendimiento asociado a esta opción es superior a «no confiscar», independientemente de lo que decidan los inversores. Los inversores se darán cuenta de esta situación y decidirán no invertir. Así, el único equilibrio Nash es el par de estrategias «no invertir» y «confiscar». Para resolver esta situación, el gobierno necesita que su estrategia «no confiscatoria» sea creíble; lo que difícilmente conseguirá si existen dudas sobre el futuro del régimen político. Supongamos ahora que el gobierno recibe de un donante extranjero una suma por valor de 201 condicionada a la adopción de políticas no confiscatorias. El juego se transforma de la siguiente manera (Diagrama 2).

${ }^{3}$ Por supuesto, hubo pocas confiscaciones de propiedades durante el primer franquismo, lo cual no impide que existiese el riesgo de tales confiscaciones en la eventualidad de que el régimen franquista fuese reemplazado. En cualquier caso, la tasa impositiva del 100 por 100 puesta como ejemplo no es sino un parámetro arbitrario más en un juego cuya finalidad principal es clarificar la exposición del argumento. 


\section{DIAGRAMA 2}

Juego entre agentes económicos privados y gobierno con recepción de ayuda (Rendimientos para los agentes económicos privados en primer lugar)

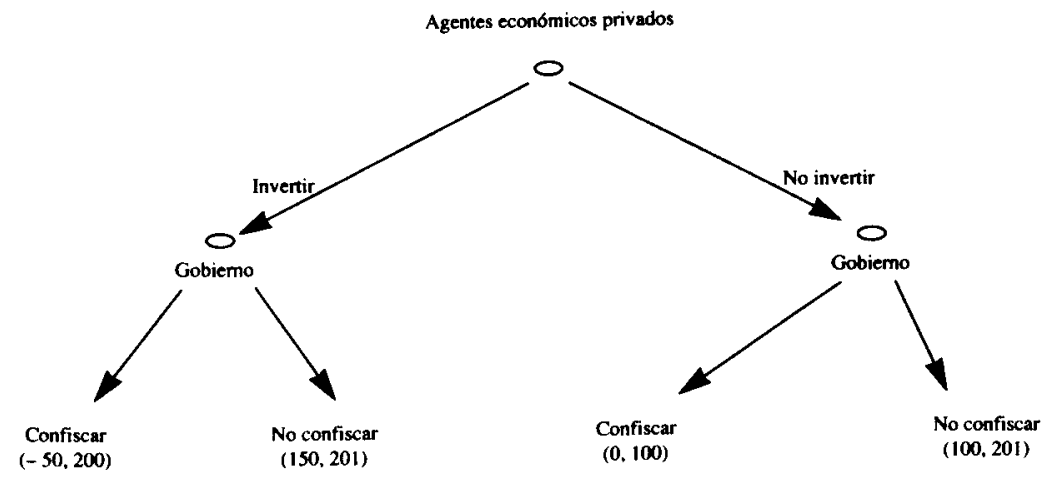

La estrategia dominante del gobierno cambia y ahora es la de «no confiscar». Pese a su crudeza, este ejemplo es revelador ${ }^{34}$. Así, no es necesariamente obvio que el resultado no dependa de las cantidades, sino del compromiso del donante - siempre y cuando los agentes perciban que el gobierno recibirá una cantidad suficiente, en nuestro ejemplo igual o mayor a 201, cuánto mayor resulta ser irrelevante. El juego enfatiza el papel necesario de la condicionalidad y así la reciente historiografía sobre el efecto del Plan Marshall se ha concentrado en examinar la influencia de los Estados Unidos en la política económica de los países receptores de dicha ayuda, explicando así cómo un programa de ayuda muy limitado en su cuantía pudo tener, sin embargo, efectos muy considerables.

Sin embargo, como hemos visto, el efecto del programa de ayuda americano sobre el cambio de política en España fue limitado, especialmente en el corto plazo. ¿Cabe aún hablar de hipótesis de credibilidad en el caso español? Para responder a este interrogante debemos reconsiderar los incentivos del donante. En palabras de The Economist: «[a]hora que los americanos tienen un interés en el país, es razonable asumir que le

${ }^{34}$ El ejercicio se realiza únicamente por su valor heurístico, espíritu con el que la teoría de juegos está marcando el análisis de las instituciones en la Historia económica. Véase North (1991), Greif (1997) y Bates et al. (1998). 
ayudarán a salir de sus dificultades económicas más serias» ${ }^{35}$. La construcción de bases militares americanas en España hacía que existiese dicho interés. Sin embargo, éste se limitaba a «proporcionar la mínima ayuda adicional que garantice la estabilidad interna en España para que así el uso de nuestras bases no se vea comprometido por desórdenes civiles» ${ }^{36}$. De ahí que la condicionalidad impuesta fuese suave ${ }^{37}$. El gobierno español había conseguido con la firma de los Acuerdos de Madrid una verdadera póliza de seguro frente a posibles inestabilidades sociopolíticas.

A diferencia de la tesis de González, quien sugiere que debido a la desregulación y liberalización se mejoraron los derechos de propiedad propiciando el crecimiento económico, en nuestra «hipótesis de credibilidad» el cambio en las expectativas no es debido principalmente a un episodio liberalizador o reformista, sino a la manera en la que los Estados Unidos se comprometen a garantizar la permanencia del régimen y con ello la estabilidad en el país ${ }^{38}$. En este sentido, es necesario enfatizar que los Pactos de Madrid no son sino la culminación de un proceso de aceptación internacional del régimen de Franco que había comenzado de manera notable con el primer crédito del Eximbank en agosto de 1950 y el retorno de embajadores a comienzos de $1951^{39}$. No se trataría tanto de que los nuevos recursos «procedentes de la ayuda americana vinieron, además, a desplazar la frontera de posibilidades de producción de la economía» como de un desplazamiento de la economía española desde el interior hacia dicha frontera ${ }^{40}$.

En los términos más simples posibles, el argumento expuesto sugiere que el programa de ayuda americano supuso una mejora de las expectativas empresariales, que tuvieron como consecuencia una mayor actividad económica e inversión privada y, por tanto, un más rápido crecimiento eco-

${ }^{35}$ The Economist, 17 de abril de 1954.

${ }^{36}$ Memorándum de la 248." reunión del Consejo de Seguridad Nacional, Washington, 12 de mayo de 1955, Foreign Relations of the United States, vol. XXVII, 1955-1957, p. 539.

${ }^{37}$ Informe sobre el desarrollo de la política de Estados Unidos hacia España, Washington, 27 de abril de 1955, Foreign Relations of the United States, vol. XXVII, 1955-1957, p. 537, y Memorándum del Vicesubsecretario de Estado para asuntos económicos al Secretario de Estado, Washington, 15 de junio de 1957, Foreign Relations of the United States, vol. IX, 1955-1957, p. 247.

${ }^{3 \times}$ Este énfasis en la estabilidad es una constante en la historiografía empírica sobre credibilidad política y crecimiento económico, véase Borner et al. (1995). González (1989/1990).

${ }^{39}$ Sobre el crédito del Eximbank, véase la monografía de Viñas (1981b). Para un tratamiento del período de ostracismo del régimen véase Portero (1989)

41) González (1989/1990), p. 30. 
nómico. Pasemos ahora a realizar una primera exploración de la información disponible que pudiese arrojar luz sobre la capacidad explicativa de este hilo argumental.

\section{UNA HIPÓTESIS DE CREDIBILIDAD (II): UNA PRIMERA APROXIMACIÓN AL REGISTRO HISTÓRICO}

No cabe dudar del papel de la inversión privada en el considerable crecimiento económico durante los años cincuenta. El capital desembolsado en sociedades en activo, que, en términos reales, había permanecido estancado durante los años cuarenta, sufre un acelerón durante los cincuenta ${ }^{41}$. La inversión privada pasó de constituir un 8,7 por 100 del gasto nacional bruto en 1941-1949 a un 15,2 por 100 en el período 1950-1958, lo que desembocó en un aumento del 51,7 por 100 del stock de capital fijo privado en términos reales durante el período 1951-1958, comparado con una reducción del mismo del 4,2 por 100 durante los cuarenta ${ }^{42}$.

Fuentes norteamericanas se refieren claramente a los «recelos del capital privado» o a la falta de confianza del mundo empresarial durante los años cuarenta y primeros cincuenta, un factor que también ha recogido la historiografía reciente ${ }^{43}$. Que, «a finales de 1953, y quizás debido a los acuerdos con Estados Unidos, observamos una nueva tendencia a construir nuevas e importantes centrales eléctricas» apunta a que el apoyo americano pudo contribuir a mejorar el clima empresarial ${ }^{44}$. En el caso al que se refiere esta cita, los elevados costes de establecimiento de grandes proyectos industriales realzan la importancia de un clima de seguridad económica y estabilidad ${ }^{45}$. Sin embargo, dada la dificultad intrínseca que supone interpretar la motivación de decisiones empresariales, los estudios cualitativos que pudiesen reclamar representatividad son extremadamente difíciles. Por su parte, a la hora de identificar el papel desempeñado por la mejora

${ }^{41}$ La tasa anual media de crecimiento aumenta desde un 1,2 por 100 en 1940-1949 hasta un 4,8 por 100 en 1950-1959. Cálculos basados en Tafunell (1989) y Prados de la Escosura (1995).

${ }^{42}$ Cubel y Palafox (1997). Los cálculos de inversión privada están basados en Carreras (1985)

${ }^{43}$ Informe de la Misión Especial de Estudio del Comité de Asuntos Exteriores de la Cámara de Representantes, diciembre 1961. En MAE, Leg. 7741, Exp. 2. Véase Fraile (1999), p. 240.

${ }^{44}$ Banco Urquijo (1954), p. 30. El párrafo hace referencia al interés del sector privado de la economía.

45 Dixit (1992). 
de la seguridad económica y la credibilidad política en explicar la inversión, los estudios de corte transversal suelen utilizar medidas subjetivas de credibilidad, basadas típicamente en la opinión de expertos sobre el nivel del riesgo-país, o bien en encuestas al empresariado sobre su percepción de la predictibilidad del gobierno ${ }^{46}$. Aunque el poder explicativo en este tipo de regresiones sea significativo, esta vía no es, obviamente, una opción en la investigación histórica. Una reciente contribución ha sido, precisamente, la elaboración de una medida objetiva de la seguridad económica, es decir, de la percepción de los agentes económicos acerca de la seguridad de sus derechos de propiedad ${ }^{47}$. Los autores de esta medida realzan que los agentes están continuamente tomando decisiones sobre en qué forma mantener sus balances monetarios. Así, determinados tipos de dinero (depósitos, etc.) requieren una mayor intensidad en el cumplimiento de los derechos de propiedad, constituyen, por tanto, dinero intensivo en contratos. Este indicador reflejará el nivel de garantías de cumplimiento de los contratos y derechos de propiedad ${ }^{48}$. El Gráfico 1 muestra la evolución de este indicador para España durante los años cincuenta.

Pese a que el indicador aumente con la intensificación del apoyo americano, este indicador no debe, sin embargo, interpretarse ligeramente. Aunque en principio la estabilidad del sistema bancario hace que tengamos confianza en que la variable no está capturando sólo el riesgo bancario, existen varios problemas en su interpretación como una medida de riesgo-país ${ }^{49}$. En primer lugar, resulta difícil evaluar hasta qué punto los incrementos son significativos. Igualmente, es éste un indicador que se verá afectado por la evolución del PIB, el grado de desarrollo del sector financiero, etc. Dado que la inflación en los años iniciales de los cincuenta es muy superior a la de mediados de la década, no es posible descartar que la evolución de este indicador venga determinada por la variación en la inflación ${ }^{50}$. Repasemos, por tanto, otras maneras alternativas de aproximarnos a la confianza empresarial.

Podemos fijarnos en los mercados financieros como un indicador de la confianza de los participantes en dichos mercados. El Gráfico 2 muestra

46 Knack y Keefer (1995).

47 Clague et al. (1999).

${ }^{48}$ Definido como $\left(\mathrm{M}_{2}-\mathrm{C}\right) / \mathrm{M}_{2}$, donde $\mathrm{M}_{2}$ es una medida amplia de la oferta monetaria y C es el efectivo en manos del público. Clague et al. (1999), p. 188.

49 Martín Aceña y Pons (1994).

so Aunque los autores del indicador afirman que la especificación es robusta a la inclusión de la inflación, resulta difícil a nivel de cada país excluir la posibilidad de que esta evolución esté marcada por otras variables (correlacionadas o no) con la seguridad económica. 


\section{GRÁFICO 1}

Indicador dinero-intensivo-en-contratos, 1949-1959

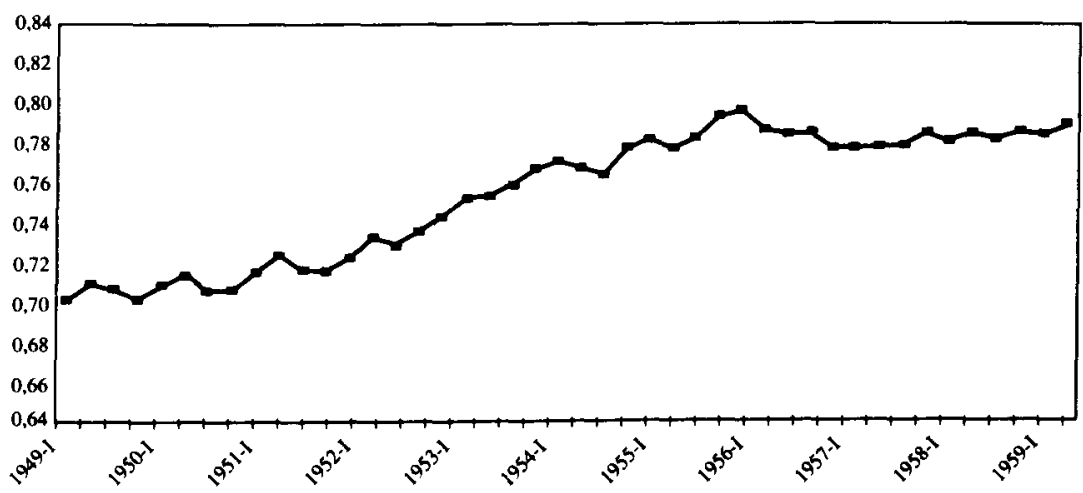

FuENTE: Cálculos basados en Martín Aceña (1989).

una continuada reducción en la prima de riesgo de la peseta en el mercado no oficial, que se mantiene particularmente estable tras 1953. El diferencial entre el tipo de cambio de Tánger y el tipo de cambio que satisface la paridad de poder adquisitivo evoluciona de manera similar, por lo que los resultados no son simplemente fruto de diferenciales de inflación en las dos zonas monetarias o de la devaluación de la peseta en 1948-1952 (al ser la estimación del tipo de cambio que satisface la paridad de poder adquisitivo independiente de los tipos de cambio oficiales).

La considerable reducción en la prima de riesgo de la peseta con anterioridad incluso a la firma del Pacto de Madrid apunta a que la mejora de las expectativas ya se venía produciendo desde el comienzo del proceso de aceptación internacional del régimen. El que la prima de riesgo (medida con el tipo de cambio PPA) alcanzase su máximo en 1950 y descendiese a partir de entonces encaja particularmente con la cronología arriba expuesta en la que el fin del aislamiento del régimen podría situarse con el primer crédito del Eximbank y el retorno de embajadores. Así pues, esta evolución no debe interpretarse como una refutación del efecto del programa de ayuda americano, pues éste comienza ya en 1950, aunque, sin duda, dificulta el identificar la contribución individual de cada uno de los muchos factores que afectaron el clima empresarial. 


\section{GRÁFICO 2}

Premio de la peseta (respecto al dólar americano) en el mercado de divisas no oficial

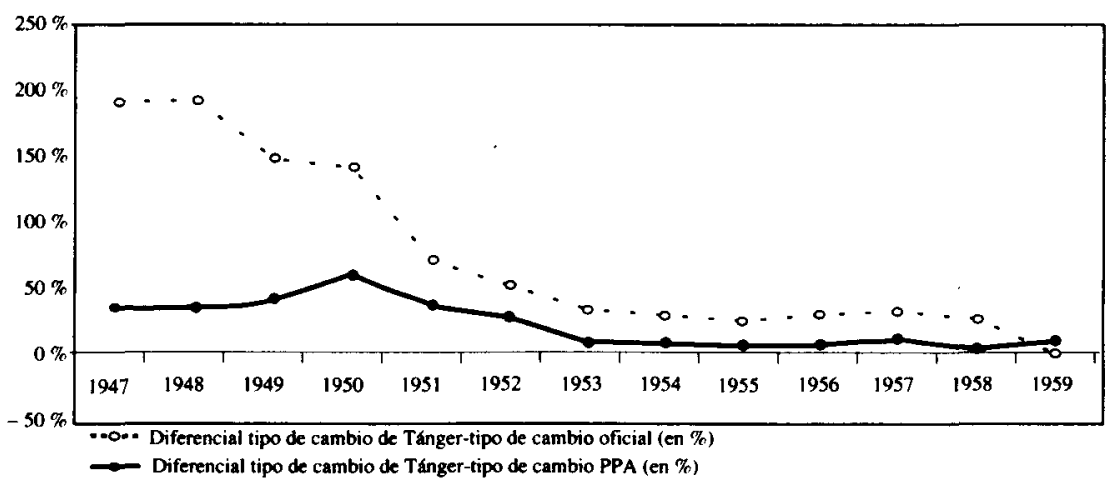

Fuentes: Tipo de cambio en Tánger en Clavera et al. (1978), p. 270; tipo de cambio oficial es el tipo de cambio medio de la balanza básica calculado por Serrano Sanz y Asensio (1997); Aixalá (1999) proporciona una estimación del tipo de cambio que satisface la paridad de poder adquisitivo (PPA).

Este breve repaso a algunos indicadores, de los muchos posibles, apunta a que sí se produjo una reacción positiva en el clima de expectativas empresariales aunque igualmente prueba lo difícil que resulta discriminar sobre las posibles causas de ésta ${ }^{51}$. Por supuesto, cuanta más importancia se dé al cambio de rumbo en la política económica en 1951 o al papel del fin de las restricciones eléctricas en determinar el clima empresarial, más relevante resulta esta cuestión.

\section{CONCLUSIÓN}

Este artículo ha explorado la visión de que el caso español se asemeja al contrafactual discutido en la literatura sobre el Plan Marshall ${ }^{52}$. Los años cuarenta españoles son ilustrativos de la extensísima intervención esta-

${ }^{51}$ Éste es precisamente el objeto de una línea de mi investigación en curso, utilizando información más detallada sobre otros mercados de activos financieros, como, por ejemplo, la Bolsa.

52 Prados de la Escosura y Sanz (1996), p. 363. 
tal en la economía y pobres resultados que quizás hubiesen asolado a Europa caso de que el Programa de Recuperación Europeo no hubiese sido llevado a cabo. Por contra, los años cincuenta españoles comparten con los países receptores de ayuda del Plan Marshall la experiencia positiva de la ayuda americana, si bien dichos efectos beneficiosos pudieron tener mecanismos de transmisión diferentes. Así, un breve repaso al efecto directo de la ayuda americana en la economía española a través de la importación de bienes con cargo a la ayuda coincide con un consenso semejante en la historiografía sobre el Plan Marshall. Sin embargo, a diferencia de lo propuesto en aquella historiografía, los posibles efectos indirectos del programa de ayuda americano en España habría que buscarlos no tanto en una influencia sustancial en el corto plazo sobre la política económica, sino en una posible mejora de la confianza empresarial, y con ella de la inversión privada. Esta interpretación tiene en común con González el énfasis en el beneficioso efecto para el funcionamiento de la economía del reforzamiento de los derechos de propiedad, pero, crucialmente, éste no es principalmente consecuencia de medidas políticas liberalizadoras o desreguladoras, sino que la mejora en las expectativas tuvo lugar como resultado de la manera en la que se articuló el apoyo americano ${ }^{53}$. Esta hipótesis podría, por tanto, reconciliar aquellas interpretaciones que resaltan el cambio que supuso la década de los cincuenta en la evolución del franquismo, y aquellas otras que enfatizan la persistencia de políticas restrictivas durante esos mismos años ${ }^{54}$. El cambio en las expectativas se traduciría en una mayor actividad económica sin haber sido necesariamente originado por un inmediato cambio reformista en la política económica. Al igual que en la historiografía sobre el Plan Marshall, la cuestión queda aquí lejos de estar resuelta, lo que no debe sino propiciar una profundización en la investigación de la hipótesis aquí apuntada.

\section{BIBLIOGRAFÍA}

AxXalí Pastó, J. (1999): La peseta y los precios, Zaragoza, Prensas Universitarias de Zaragoza.

Anderson, C. W. (1970): The Political Economy of Modern Spain. Policy Making in an Authoritarian System, Madison, University of Wisconsin Press.

AsEnSIO, M. J. (1995): «El proceso de apertura exterior de los cincuenta y el arancel de 1960», tesis doctoral, Universidad de Zaragoza.

"3 González (1989/1990).

${ }^{4}$ García Delgado (1986); Gómez Mendoza y San Román (1997) y San Román (1999). 
Baldrich, A. (1957): «Balance y Efectos Económicos de la Ayuda Norteamericana», Moneda y Crédito, 61, junio, pp. 27-46.

Banco Mundial (1998): Assessing Aid: What Works, What Doesn't and Why?, Washington, D. C., Oxford University Press.

Banco Urquijo. Servicio de Estudios (1954): La economía española, 1952-53, Madrid.

BAtES, R., et al. (1998): Analytic Narratives, Princeton, NJ, Princeton University Press.

Berger, H., y Ritschl, A. (1995): «Germany and the Political Economy of the Marshall Plan, 1947-52: A Re-revisionist View», en B. EICHENGREEN (ed.), Europe's Postwar Recovery, Cambridge, UK Cambridge University Press, pp. 199.245.

Borner, S.; Brunetti, A., y Weder, B. (1995): Political Credibility and Economic Development, New York, St Martin's Press.

BuEsA, M. (1984): «Las restricciones a la libertad de industria en la política industrial española (1938-1963)», Información Comercial Española, 606, pp. 107-121.

CARreras, A. (1985): «Gasto Nacional Bruto y Formación de Capital en España, 1849-1958: primer ensayo de estimación», en L. PrADOS DE LA EscosurA y P. MARTín ACEÑa (eds.), La nueva historia económica en España, Madrid, Tecnos, pp. 17-51.

Clague, C.; Keefer, P.; Knack, S., y Olson, M. (1999): «Contract-Intensive Money: Contract Enforcement, Property Rights, and Economic Performance», Journal of Economic Growth, 4, 2, pp. 185-211.

Clavera, J.; Esteban, J. M.; Monos, M. A.; Montserrat, A., y Ros Hombravella, J. (1978): Capitalismo español: de la autarquía a la estabilización (1939-1959), Madrid, Edicusa, 2. ${ }^{\mathrm{a}}$ ed.

Cubel, A., y Palafox, J. (1997): «El stock de capital de la economía española, 1900-1958», Revista de Historia Industrial, 17, pp. 113-146.

De Blors, E. N. (1967): 12 Years of Achievement Under Public Law 480, Washington, D. C., US Department of Agriculture.

De Long, B., y Eichengreen, B. (1993): «The Marshall Plan: History's Most Successful Structural Adjustment Program», en R. Dornbusch, W. Nölling y R. LAYARD (eds.), Postwar Economic Reconstruction and Lessons for the East Today, Cambridge, MA, MIT Press, pp. 189-230.

DixIt, A. K. (1992): «Investment and Hysteresis», Joumal of Economic Perspectives, 6, 1, pp. 107-132.

Dixtr, A. K., y Nalebuff, B. J. (1991): Thinking Strategically, New York, Norton.

EDWARDS, J. (1999): Anglo-American Relations and the Franco Question, 1945-1955, Oxford, Clarendon Press.

Eichengreen, B. (1993): Reconstructing Europe's Trade and Payments. The European Payments Union, Manchester, Manchester University Press.

- (1996): «Institutions and Economic Growth», en N. F. R. CrafTs y G. Toniolo (eds), Economic Growth in Europe Since 1945, Cambridge, UK, Cambridge University Press.

Eichengreen, B., y UzAN, M. (1992): «The Marshall Plan: Economic Effects and Implications for Eastern Europe and the Former USSR», Economic Policy, 14 , pp. 14-75. 
Esposrro, C. (1994): America's Feeble Weapon: Funding the Marshall Plan in France and Italy, 1948-1950, Westport, Conn., Greenwood Press.

FAnjul, E. (1981): «El papel de la ayuda americana en la economía española», Información Comercial Española, 577, septiembre, pp. 159-166.

Fernández dE VALDERrama, G. (1964): «España-USA, 1953-1964», Economía Financiera, 6, pp. 14-51.

Fontana, J., y Nadal, J. (1976): «Spain, 1914-1970», en C. M. Cipolla (ed.), The Fontana Economic History of Europe. Contemporary economies, vol. 6-2, Glasgow, William Collins Sons, pp. 460-529.

Fralle Balbín, P. (1999): «Industrial Policy under Authoritarian Politics: the Spanish Case», en J. Foreman-PeCK y G. Federico (eds)., European Industrial Policy, Oxford, Oxford University Press, pp. 233-267.

Fusi AuzPurúA, J. P. (1995): Franco, Madrid, Taurus.

García Delgado, J. L. (1986): «Crecimiento industrial y cambio en la política española en el decenio de 1950. Guía para un análisis», Hacienda Pública Española, 100, pp. 287-296.

Gomez MendozA, A., y SAN Román, E. (1997): «Competition between Private and Public Enterprise in Spain, 1939-1959: An Alternative View», Business and Economic History, 26, 2, pp. 696-708.

GonzÁLEZ, M. J. (1979): La economia política del franquismo (1940-1970), Madrid, Tecnos.

- (1989/1990): «La autarquía económica bajo el régimen del General Franco: una visión desde la teoría de los derechos de propiedad», Información Comercial Española, 676-677, pp. 19-31.

GrEIF, A. (1997): «Microtheory and Recent Developments in the Study of Economic Institutions through Economic History», en D. KREPS y K. WALLIS (eds.), Advances in Economics and Econometrics, vol. 2, Cambridge, UK, Cambridge University Press, pp. 79-113.

GuiraO, F. (1996): «The United States, Franco, and the Integration of Europe», en F. H. Heller y J. R. Gillingham (eds.), The United States and the Integration of Europe: Legacies of a Postwar Era, New York, St Martin's Press.

Harrison, J. (1978): An Economic History of Modern Spain, New York, Holmes and Meier.

- (1985): The Spanish Economy in the Twentieth Century, London, Croom Helm.

InstTTUTO DE Estudios POLfTicos (1958): La estructura de la economía española. Tablas input-output, Madrid.

JARQUE ÍñigueZ, A. (1998): Queremos esas bases, Alcalá de Henares, Universidad

de Alcalá.
KNACK, S., y KEEFER, P. (1995): «Institutions and Economic Performance: Cross-Country Tests Using Alternative Institutional Measures», Economics and Politics, 7, 3, pp. 207-227.

LIEDTKE, B. N. (1998): Embracing a Dictatorship. US Relations with Spain, 1945-53,

Houndmills, McMillan Press.
- (1999): «Spain and the United States, 1945-1975» en P. Preston y S. BaLFour (eds.), Spain and the Great Powers in the Twentieth Century, New York, Routledge, pp. 229-244. 
Martin ACEÑA, P. (1989): «Una estimación de los principales agregados monetarios en España 1940-1962», Madrid, Banco de España.

Martín Aceña, P., y Pons, M. A. (1994): «Spanish Banking after the Civil War, 1940-1962», Financial History Review, 1, 2, pp. 121-138.

MARTiNEz RuIz, E. (1998): «Las balanzas de pagos de la autarquía. Una revisión», Working Paper, 98-23, Universidad Carlos III.

Milward, A. S. (1984): The Reconstruction of Western Europe, 1945-1951, London, Methuen.

NORTH, D. C. (1991): «Institutions», Joumal of Economic Perspectives, 5, pp. 97-112.

PORTERO, F. (1989): Franco aislado: la cuestión española (1945-1950), Madrid, Aguilar.

Prados de la Escosura, L. (1995): «Spain's Gross Domestic Product, 1850-1993: Quantitative Conjectures. Appendix», Working Paper, 95-06, Universidad Carlos III.

Prados de la Escosura, L., y SANZ, J. C. (1996): «Growth and Macroeconomic Performance in Spain, 1939-1993», en N. F. R. CRAFTS y G. Toniolo (eds.), Economic Growth in Europe Since 1945, Cambridge, UK, Cambridge University Press, pp. 355-387.

Preston, P. (1993): Franco: A Biography, London, Harper Collins.

Rovira SÁnchez-Herrero, J. J. (1960): «La ayuda estadounidense», en Centro de Estudios Tributarios, Las inversiones de capital extranjero en España, vol. I., Madrid, AGESA, pp. 114-166.

Rubottom, R. R., y CARTER MurPhy, J. (1984): Spain and the United States since World War II, New York, Praeger Publishers.

SÁinz Moreno, F. (1965): «Historia de las inversiones extranjeras en España», Boletín de Estudios Económicos, XX, 65, pp. 373-408.

SAN ROMÁN, E. (1999): Ejército e Industria: el nacimiento del INI, Barcelona, Crítica.

SARDÁ, J. (1978): «Prólogo» a J. ClAVERA, et al., Capitalismo español: de la autarquía a la estabilización (1939-1959), Madrid, Edicusa.

SAUSE, O. L. (1957): «Algunos aspectos de los programas de cooperación económica hispano-norteamericanos», Revista de Economía Política, 8, 1, pp. 35-45.

Serrano Sanz, J. M., y Asensio CASTHLO, M. J. (1997): «El ingenierismo cambiario. La peseta en los años del cambio múltiple, 1948-1959», Revista de Historia Económica, 15, 3, pp. 545-573.

SHEARER, E. B. (1959): «Significado para España de la ayuda económica norteamericana», Revista de Economía Política, 10, 3, pp. 989-1006.

SPITÄLlER, E., y GALY, M. (1992): «Spain: Landmarks in Economic Development, 1939-92», Working paper, 92-78, IMF.

STANFORD RESEARCH INSTTUUTE (1972): American Investments in Spain, Barcelona, American Chamber of Commerce in Spain.

TACCONI, S. (1955): «Acuerdos y convenios de España con los Estados Unidos de América», De Economía, 8, 37-38, pp. 601-635.

TAFunEll, X. (1989): «Asociación mercantil y Bolsa», en A. Carreras (ed.), Estadísticas históricas de España. Siglos XIX.XX, Madrid, Fundación Banco Exterior, pp. 461-494.

Tamames, R. (1986): La República. La Era de Franco, Madrid, Alianza. 
Tena, A. (1989): «Comercio exterior», en A. CARRERAs (ed.), Estadísticas históricas de España. Siglos XIX-XX, Madrid, Fundación Banco Exterior, pp. 327-362.

VIÑAS, A. (1981a): Los pactos secretos de Franco con Estados Unidos: bases, ayuda económica, recortes de soberanía, Barcelona, Grijalbo.

- (1981b): «La primera ayuda económica norteamericana a España», Lecturas de economía española e internacional: homenaje al cincuenta aniversario del cuerpo de Técnicos Comerciales del Estado, Madrid, Ministerio de Comercio.

Viñas, A.; Viñuela, J. V.; Eguidazu, F.; Fernández Pulgar, C., y Florensa, S. (1979): Política comercial exterior en España (1931-1975), vol. 2, Madrid, Banco Exterior de España.

Yotopoulos, P. A., y Nugent, J. (1973): «A Balanced Growth Version of the Linkage Hypothesis: A Test», Quarterly Joumal of Economics, 87, 2, pp. 157-171.

\section{FUENTES}

Foreign Relations of the United States, United States Government Printing Office, Washington, D. C.

Ministerio de Asuntos Exeriores (MAE), Madrid, Archivo Histórico, Serie Renovada.

\section{Publicaciones OfiCiales}

Banco de España: Informe sobre la evolución de la economía española, varios números, Madrid.

Dirección General de Cooperación Económica: Informe sobre el desarrollo de la ayuda económica, varios números, Madrid.

Export-Import Bank of the US: Semiannual Report to Congress, varios números, Washington, D. C.

US Agency for International Development (1963): US Overseas Loans and Grants from Intemational Organizations, July 1, 1945-June 30, 1963, Washington, D. C. 